\title{
A new effective Lagrangian for nuclear matter
}

\author{
T.S. Biró and J. Zimányi \\ MTA KFKI Research Institute for Particle and Nuclear Physics \\ Budapest 114., P.O.Box 49, H-1525 Hungary
}

\begin{abstract}
The relativistic mean field model, the Zimányi - Moszkowski (ZM) Lagrangian describes nuclear matter and stable finite nuclei even in the non-relativistic limit. It fails, however, to predict the correct non-relativistic spin-orbit (SO) coupling. In this paper we improve on this matter by an additional tensor coupling analogous to the anomalous gyromagnetic ratio. It can be adjusted to describe the SO-term without changing the mean field solution of the ZM-Lagrangian for nuclear matter.
\end{abstract}

${ }^{1}$ jzimanyi@sunserv.kfki.hu 


\section{Introduction}

The advantages of describing nuclear physics relativistically are to find connections between different parts of the non-relativistic mean field nuclear potential, such as the linear energy dependence emerging from kinematic features of an effective Dirac equation for single nucleons or a mild mean potential being balanced between strong attraction and repulsion.

The most known such model, the Walecka model[1], 2] was first intended to be an elementary description, but it was soon discovered that only an effective Lagrangian approach is fruitful. Renormalization problems and divergences of higher meson loop corrections with a strong effective coupling revealed that by dropping the requirement of an elementary approach a higher degree of freedom in the choice of the effective Lagrangian can be exploited.

The Walecka model describes the non-relativistic mean field potential, $(V-S)$, and spinorbit coupling $\frac{d}{d r}(V+S)$, both derived from the same effective Dirac equation. Unfortunately it fails to describe compressibility and a finite nucleus, which remains bound in a quantum molecular dynamics (QMD) approach. Simulation of dynamics including heavy ions reactions at relativistic energies, however, desperately needs a Lagrangian, which is relativistic and has a stable nucleus. Such an effective Lagrangian was proposed by Zimányi and Moszkowski [3] recently. It works with less repulsion, $V$, with an attractive mean field, $S$ which is bounded from above by the free nucleon mass, $M$ and describes stable finite nuclei[4]-[8]. Up to now only the too weak SO-potential was a manque of this model[9].

Recent investigations did show that this Lagrangian has many advantageous properties in describing the bulk properties of the nuclear matter [10]-12]. The optical potential derived from the ZM Lagrangian also agrees with experimental findings 13. Finite nuclei described within the framework of this model are used in QMD calculations 14] as well.

In this letter we show that an additional tensor term influences the spin - orbit part of the non-relativistic potential without spoiling the benevolent property of the ZM Lagrangian: the mild repulsive mean field. This reduced repulsion is necessary to describe stable finite nuclei in the quantum molecular dynamical approach to heavy ion collisions. Throughout this paper we use Minkowskian metric with the signature $(+,-,-,-)$ and the convention 
$\hbar=c=1$.

Improved effective Lagrangian

The Zimányi-Moszkowski (ZM) Lagrangian, $\mathcal{L}_{\mathrm{ZM}}$, is formulated in terms of nucleon fields $\psi$, a scalar $\sigma$ and vector $\omega_{\mu}$ mean fields. The corresponding scalar and vector coupling constants are $g_{s}$ and $g_{v}$, respectively. Now we add a tensor term to this Lagrangian which couples the antisymmetric spin current density $\bar{\psi} \sigma_{\mu \nu} \psi$ to the field strength of the vector field $F^{\mu \nu}$

$$
\begin{aligned}
\mathcal{L}_{\mathrm{ZMB}}= & -\bar{\psi} M_{*} \psi+\bar{\psi}\left(\left(i \partial_{\mu}-g_{v} \omega_{\mu}\right) \cdot \gamma^{\mu}+\frac{\lambda}{2 M_{*}} g_{v} \sigma_{\mu \nu} F^{\mu \nu}\right) \psi \\
& -\frac{1}{4} F_{\mu \nu} F^{\mu \nu}+\frac{1}{2} m^{2} \omega_{\mu} \omega^{\mu}+\frac{1}{2} \partial_{\mu} \sigma \cdot \partial^{\mu} \sigma-U(\sigma) .
\end{aligned}
$$

with

$$
M_{*}=\frac{M}{1+g_{s} \sigma / M}
$$

being the effective nucleon mass. $M$ and $m$ are the bare nucleon and vector meson masses, respectively, and $U(\sigma)$ includes the $\frac{1}{2} m_{\sigma}^{2} \sigma^{2}$ mass term and an eventual self interaction.

In order to deal with static mean fields over a finite spatial extension we assume that only $\sigma$ and $\omega_{\mu}=\left(\omega_{0}, \overrightarrow{0}\right)$ differs from zero and all time derivative vanishes. In this description of static nuclei $\omega_{0}$ and $\sigma$ may have gradients, however. The field strength $F_{\mu \nu}$ has only electric components $F_{0 k}=-\nabla_{k} \omega_{0}$. The corresponding coefficient Dirac-matrix in the tensor term is $\sigma_{0 k}=-i \alpha_{k}$. The equations of motion for the meson fields can be derived from the improved Lagrangian easily:

$$
\begin{aligned}
-\square \sigma-U^{\prime}(\sigma)+\frac{M_{*}^{2}}{M^{2}} g_{s} \bar{\psi} \psi+\frac{\lambda}{2 M} \frac{g_{s}}{M} g_{v} F^{\mu \nu} \bar{\psi} \sigma_{\mu \nu} \psi & =0, \\
\partial_{\mu}\left(F^{\mu \nu}+\frac{\lambda}{M_{*}} g_{v} \bar{\psi} \sigma_{\mu \nu} \psi\right)+m^{2} \omega^{\nu}-g_{v} \bar{\psi} \gamma^{\nu} \psi & =0 .
\end{aligned}
$$

These equations lead to the same mean field equations as without the tensor term for all unpolarized media with $\left\langle\bar{\psi} \sigma_{\mu \nu} \psi\right\rangle=0$. Using this the homogeneous static mean field solution arises as in the case of the Zimányi - Moszkowski Lagrangian with $U(\sigma)=\frac{1}{2} m_{\sigma}^{2} \sigma^{2}$ :

$$
\begin{aligned}
m_{\sigma}^{2} \sigma & =\frac{M_{*}^{2}}{M^{2}} g_{s}\langle\bar{\psi} \psi\rangle, \\
m^{2} \omega_{0} & =g_{v}\left\langle\psi^{\dagger} \psi\right\rangle .
\end{aligned}
$$


The Dirac equation derived from the above Lagrangian on the other hand has a new contribution

$$
\left(\gamma^{\mu}\left(i \partial_{\mu}-g_{v} \omega_{\mu}\right)+\frac{\lambda}{2 M_{*}} g_{v} \sigma_{\mu \nu} F^{\mu \nu}-M_{*}\right) \psi=0
$$

In order to investigate the non-relativistic limit we consider the Hamiltonian, expressing $\hat{H} \psi=i \partial_{t} \psi$ from the above equation. Multiplication with $\gamma_{0}$ from the left and neglecting vanishing mean field and time derivative components gives the relativistic Hamiltonian

$$
\hat{H}=-i \vec{\alpha} \vec{\nabla}+g_{v} \omega_{0}-\frac{\lambda}{2 M_{*}} i \vec{\gamma} \vec{\nabla} g_{v} \omega_{0}+M_{*} \beta
$$

where we used the Dirac representation of matrices

$$
\beta=\left(\begin{array}{cc}
1 & 0 \\
0 & 1
\end{array}\right), \quad \vec{\alpha}=\left(\begin{array}{cc}
0 & \vec{\sigma} \\
\vec{\sigma} & 0
\end{array}\right), \quad \vec{\gamma}=\left(\begin{array}{cc}
0 & \vec{\sigma} \\
-\vec{\sigma} & 0
\end{array}\right) .
$$

For the sake of a more compact notation we introduce $S=g_{s} \sigma /\left(1+g_{s} \sigma / M\right)$ and $V=g_{v} \omega_{0}$. This notation automatically satisfies the relation $M_{*}=M-S$. The relativistic Hamiltonian equals

$$
\hat{H}=\beta M^{*}+\vec{\alpha} \vec{p}+V-i \frac{\lambda}{2 M_{*}} \vec{\gamma}(\vec{\nabla} V) .
$$

where the momentum operator $\vec{p}=-i \vec{\nabla}$ is introduced. The Dirac matrices and spinors in $2 \times 2$ notation, $\psi=(\varphi, \chi)$, lead to coupled equations for the upper $(\varphi)$ and lower $(\chi)$ components. The non-relativistic Hamiltonian is the effective Hamiltonian acting on the upper component of the Dirac spinor. We get the following eigenvalue equations

$$
\begin{aligned}
& \left(V+M^{*}-E\right) \varphi+\vec{\sigma}\left(\vec{p}-i \frac{\lambda}{2 M_{*}} \vec{\nabla} V\right) \chi=0 \\
& \vec{\sigma}\left(\vec{p}+i \frac{\lambda}{2 M_{*}} \vec{\nabla} V\right) \varphi+\left(V-M^{*}-E\right) \chi=0
\end{aligned}
$$

with $E$ being the relativistic energy eigenvalue. The non-relativistic energy eigenvalue is therefore $e=E-M$. Introducing further simplifying notations,

$$
\vec{p}_{ \pm}=\vec{p} \mp i \frac{\lambda}{2 M_{*}}(\vec{\nabla} V), \quad V_{ \pm}=V \pm M^{*}-E
$$

it reduces to

$$
\begin{aligned}
V_{+} \varphi+\vec{\sigma} \vec{p}_{+} \chi & =0 \\
\vec{\sigma} \vec{p}_{-}+V_{-} \chi & =0 .
\end{aligned}
$$


Here $V_{ \pm}$can be expressed by using the non-relativistic energy eigenvalue as $V_{+}=V-S-e$ and $V_{-}=V-S-e-2 M_{*}$. Extracting now $\chi$ from the second equation and substituting back into the first gives

$$
\left(V_{+}-\vec{\sigma} \vec{p}_{+} \frac{1}{V_{-}} \vec{\sigma} \vec{p}_{-}\right) \varphi=0 .
$$

A non-relativistic Hamiltonian can be obtained by expressing the energy eigenvalue $e$ from this equation. We get

$$
\hat{h} \varphi=e \varphi=\left(V-S-\vec{\sigma} \vec{p}_{+} \frac{1}{V_{-}} \vec{\sigma} \vec{p}_{-}\right) \varphi
$$

The non-relativistic expansion of this operator together with a gradient expansion for the mean field factors $V$ and $S$ leads to the non-relativistic nuclear potential. The spin-orbit coupling term arises as a sub-leading correction in an $\mathcal{O}\left(1 / M_{*}\right)$ expansion. Traditionally it is due to the fact that the $\vec{p}$ gradient operator does not commute with $1 / V_{-}$. In the present case we have an additional contribution to this term stemming from the tensor coupling due to $\vec{p}_{+} \times \vec{p}_{-} \neq 0$. We proceed by calculating

$$
\vec{p}_{+} \frac{1}{V_{-}}=\frac{1}{V_{-}} \vec{p}_{+}-i \vec{\nabla} \frac{1}{V_{-}}
$$

After noting that $\vec{\nabla} V_{-}=\vec{\nabla}(V+S)$ both terms on the right hand side can be expanded in inverse powers of the effective mass. We get

$$
\vec{p}_{+} \frac{1}{V_{-}}=-\frac{\vec{p}}{2 M_{*}}+\frac{1}{4 M_{*}^{2}}([e-(V-S)] \vec{p}+i \vec{\nabla}[(1+\lambda) V+S])+\ldots
$$

Let us denote this result by $-\vec{\pi}_{+} / 2 M_{*}$. Using this notation the non-relativistic Hamiltonian is

$$
\hat{h}=V-S+\frac{1}{2 M_{*}} \vec{\sigma} \cdot \vec{\pi}_{+} \vec{\sigma} \cdot \vec{p}_{-}+\ldots
$$

with

$$
\vec{\pi}_{+}=\vec{p}-\frac{1}{2 M_{*}}([e-(V-S)] \vec{p}+i \vec{\nabla}[(1+\lambda) V+S])
$$

Now we utilize the general algebraic identity,

$$
\vec{\sigma} \cdot \vec{\pi}_{+} \quad \vec{\sigma} \cdot \vec{p}_{-}=\vec{\pi}_{+} \cdot \vec{p}_{-}+i \vec{\sigma}\left(\vec{\pi}_{+} \times \vec{p}_{-}\right)
$$

The individual terms are again expanded

$$
\vec{\pi}_{+} \cdot \vec{p}_{-}=\left(1-\frac{e-(V-S)}{2 M_{*}}\right) \vec{p}^{2}-\frac{1}{2 M_{*}} \vec{\nabla}(V+S) \cdot \vec{\nabla}+\vec{\nabla}\left(\frac{\lambda}{2 M_{*}} \vec{\nabla} V\right)+\ldots
$$


and

$$
\vec{\pi}_{+} \times \vec{p}_{-}=\frac{-i}{2 M_{*}} \vec{\nabla}[(1+2 \lambda) V+S] \times \vec{p}+\vec{\nabla} \frac{\lambda}{2 M_{*}} \times \vec{\nabla} V+\ldots
$$

The non-relativistic energy eigenvalue of (16) in an $1 / M_{*}$ is given by

$$
\hat{h}_{0}=V-S+\frac{\vec{p}^{2}}{2 M_{*}}+\ldots
$$

Substituting this leading order result into the coefficient of $\vec{p}^{2}$ in the next order term we make a higher order error only. This arises as a relativistic correction to the kinetic energy

$$
\hat{h}_{1}=-\frac{1}{2 M_{*}} \frac{e_{0}-(V-S)}{2 M_{*}} \vec{p}^{2}=-\frac{\vec{p}^{4}}{8 M_{*}^{3}}+\ldots
$$

Two further interesting term arise from the scalar product $\vec{\pi}_{+} \cdot \vec{p}_{-}$:

$$
\hat{h}_{2}=-\frac{1}{4 M_{*}^{2}} \vec{\nabla}(V+S) \cdot \vec{\nabla}+\frac{1}{2 M_{*}} \vec{\nabla}\left(\frac{\lambda}{2 M_{*}}\right) \vec{\nabla} V .
$$

Factorizing the $1 / 4 M_{*}^{2}$ coefficient we get

$$
\hat{h}_{2}=-\frac{1}{4 M_{*}^{2}}\left(\vec{\nabla}(V+S) \cdot \vec{\nabla}-\lambda M_{*} \vec{\nabla}\left(\frac{1}{M_{*}} \vec{\nabla} V\right)\right) .
$$

This will be the analogue of the so-called Darwin term. After symmetrization and integration by part we arrive at

$$
e_{2}=\frac{1}{8 M_{*}^{2}} \vec{\nabla}^{2}[(1+2 \lambda) V+S]+\frac{1}{4 M_{*}^{3}}(1+\lambda) \vec{\nabla} S \cdot \vec{\nabla}(V+S)
$$

The familiar non relativistic Darwin term stems from a Coulomb potential $\left(V=V_{C}, S=0\right.$ and $\left.M_{*}=M\right)$. In this case $\vec{\nabla}^{2} V_{C} \propto \delta(\vec{r})$ and it can be interpreted as the spin-orbit energy shift to zero angular momentum states.

Finally the term stemming from the vector product between $\vec{\pi}_{+}$and $\vec{p}_{-}$leads to

$$
\hat{h}_{3}=\frac{1}{4 M_{*}^{2}} \vec{\sigma}(\vec{\nabla}((1+2 \lambda) V+S) \times \vec{p})+i \vec{\sigma} \frac{1}{2 M_{*}}\left(\vec{\nabla} \frac{\lambda}{2 M_{*}} \times \vec{\nabla} V\right) .
$$

Acting by the gradient $\vec{\nabla}$ on the $1 / M_{*}$ coefficient a result higher order in $1 / M_{*}$ arises, which can be consequently neglected. Finally we arrive at

$$
\hat{h}_{3}=\frac{1}{4 M_{*}^{2}} \vec{\sigma}(\vec{\nabla}((1+2 \lambda) V+S) \times \vec{p})+\ldots
$$

This result - and its derivation - is analogous to the treatment of an anomalous gyromagnetic ratio $\left(\lambda=\Delta g / 4, g_{v}=e\right)$, [15]. 
For central mean fields $V=V(r), S=S(r)$ the first term of this expression can be rewritten by using the angular momentum operator, $\vec{L}=\vec{r} \times \vec{p}$ :

$$
\hat{h}_{3}=\frac{1}{4 M_{*}^{2}}\left(\frac{1}{r} \frac{d}{d r}((1+2 \lambda) V+S)\right) \vec{\sigma} \cdot \vec{L} .
$$

\section{Conclusion}

Summarizing the main effect of introducing the direct tensor coupling term in the relativistic Lagrangian is to enhance the role of the vector mean field $V$ to $(1+2 \lambda) V$ in leading order spin-orbit corrections to the non-relativistic single nucleon energy. From this result we conclude that the effective strength of the spin-orbit coupling is increased by the new term in the Lagrangian, $V_{S O}=(1+2 \lambda) V+S$. This is the main result of this paper.

The equations of motion for the mean fields do include an additional term due to the tensor coupling. This has no effect, however, in the mean field approximation to unpolarized media. Therefore estimating the spin-orbit potential we can use the values of $V$ and $S$ obtained self consistently for the ZM Lagrangian $(V=82 \mathrm{MeV}, S=140 \mathrm{MeV})$. Comparing the strength of the respective terms with that of the Walecka model $(V+S=785 \mathrm{MeV})$ we obtain $\lambda=3.5$. One can improve on this estimate by applying the presently described Lagrangian, $\mathcal{L}_{\mathrm{ZMB}}$, for finite nuclei.

It is interesting to note that P.G. Reinhard and his collaborators also considered a tensorial coupling in connection with the original Walecka Lagrangian. They discarded, however, this possibility in that case, because they found its contribution negligible[16].

\section{Acknowledgment}

The drilling questions and steady requiring of Judit Németh and S. Moszkowski, which triggered this work, are gratefully acknowledged. This work was supported by the Hungarian Science Research Fund, OTKA No.T014213. 


\section{References}

[1] J.D. Walecka, Ann. Phys. 83 (1974) 491

[2] S.A. Chin, J.D. Walecka, Phys. Lett. B52 (1974) 24

[3] J.Zimányi, S. Moszkowski, Phys. Rev. C42 (1990) 1416

[4] N.K. Glendenning, F. Weber, S.A. Moszkowski, Phys. Rev. C45 (1992) 844

[5] N.K. Glendenning, Phys.Rev. D46 (1992) 1274

[6] K. Miyazaki, Prog.Theor.Phys. 91 (1994) 1271

[7] K. Miyazaki, Prog.Theor.Phys. 93 (1995) 137

[8] M. Prakash, J.R. Cooke, J.M. Lattimer, Phys. Rev. D52 (1995) 661

[9] S.A. Moszkowski, private communication

[10] A. Delfino, C.T. Coelko, M. Malkeiro, Phys. Lett. B345 (1995) 361

[11] A. Bhattacharyya, S. Raha, Phys. Rev. C53 (1996) 522

[12] R. Aguirre, O. Civitarese, A.L. de Paoli, Nucl. Phys. A597 (1996) 543

[13] H. Feldmeier, J. Lindner, Z. Phys. A341 (1991) 83

[14] H. Feldmeier, J.Németh, G. Papp, GSI-Preprint 95-47, nucl-th/9508028, submitted to Heavy Ion Physics

[15] C. Itzykson, J.-B. Zuber, Quantum Field Theory, McGraw-Hill, New York, 1985

[16] P.G. Reinhard, Rep. Prog. Phys. 52 (1989) 439 